\title{
Aggregation of PEGylated liposomes driven by hydrophobic forces
}

\author{
Tamás Bozó ${ }^{\mathrm{a}, *, 1}$, Tamás Mészáros ${ }^{\mathrm{b}, \mathrm{c}, 1}$, Judith Mihály $^{\mathrm{d}}$, Attila Bóta ${ }^{\mathrm{d}}$, \\ Miklós S.Z. Kellermayer ${ }^{\mathrm{a}, \mathrm{e}}$, János Szebeni ${ }^{\mathrm{b}, \mathrm{c}}$, Benedek Kálmán ${ }^{\mathrm{b}}$ \\ a Department of Biophysics and Radiation Biology, Semmelweis University, 1094 Budapest, Tüzoltó Str. 37-47, Hungary \\ ${ }^{\mathrm{b}}$ Nanomedicine Research and Education Center, Semmelweis University, 1089 Budapest, Nagyvárad Square 4, Hungary \\ c SeroScience Ltd., 1089 Budapest, Nagyvárad Square 4, Hungary \\ ${ }^{\mathrm{d}}$ Biological Nanochemistry Research Group, Institute of Materials and Environmental Chemistry, Research Centre for Natural Sciences, Hungarian Academy \\ of Sciences, 1117 Budapest, Magyar tudósok körútja 2, Hungary \\ e MTA-SE Molecular Biophysics Research Group, Semmelweis University, 1094 Budapest, Tủzoltó Str. 37-47, Hungary
}

\section{A R T I C L E I N F O}

\section{Article history:}

Received 4 November 2015

Received in revised form 23 June 2016

Accepted 27 June 2016

Available online 28 June 2016

Dedicated to the memory of Berci (Benedek Kálmán), a great colleague and friend.

\section{Keywords:}

Liposome

PEG

Aggregation

Fusion

Hydrophobic effect

Ammonium sulfate

Kosmotropic salt

\begin{abstract}
A B S T R A C T
Polyethylene glycol (PEG) is widely used to sterically stabilize liposomes and improve the pharmacokinetic profile of drugs, peptides and nanoparticles. Here we report that ammonium sulfate (AS) can evoke the aggregation of PEGylated vesicles in a concentration-dependent manner. Liposomes with 5 mol\% PEG were colloidally stable at AS concentrations up to $0.7 \mathrm{mM}$, above which they precipitated and formed micron-size aggregates with irregular shape. While aggregation was reversible up to $0.9 \mathrm{M}$ of AS, above $1 \mathrm{M}$ fusion occurred, which irreversibly distorted the size distribution. Zeta potential of liposomes markedly increased from $-71 \pm 2.5 \mathrm{mV}$ to $2 \pm 0.5 \mathrm{mV}$ upon raising the AS concentration from 0 to $0.1 \mathrm{M}$, but no considerable increase was seen during further AS addition, showing that the aggregation is independent of surface charge. There was no aggregation in the absence of the PEG chains, and increasing PEG molar\% shifted the aggregation threshold to lower AS concentrations. Changes in the FTIR spectral features of PEGylated vesicles suggest that AS dehydrates PEG chains. Other kosmotropic salts also led to aggregation, while chaotropic salts did not, which indicates a general kosmotropic phenomenon. The driving force behind aggregation is likely to be the hydrophobic effect due to salting out the polymer similarly to what happens during protein purification or Hydrophobic Interaction Chromatography. Since liposome aggregation and fusion may result in difficulties during formulation and adverse reaction upon application, the phenomena detailed in this paper may have both technological and therapeutical consequences.
\end{abstract}

(C) 2016 Elsevier B.V. All rights reserved.

\section{Introduction}

Polyethylene glycol (PEG), a linear polymer of $\left[-\mathrm{CH}_{2}-\mathrm{CH}_{2}-\mathrm{O}\right]$ units, is commonly used in drug delivery to modify pharmacokinetic properties of active agents. Attaching PEG chains to small molecules, peptides, proteins, oligonucleotides or nanoparticles may effectively reduce their enzymatic degradation and prolong their circulation time in blood [1]. An important example is liposome PEGylation, which provides "stealth" characteristics to liposomes helping them to evade phagocytosis by macrophages,

\footnotetext{
* Corresponding author.

E-mail addresses: bozo.tamas@med.semmelweis-univ.hu (T. Bozó), tmeszaros@seroscience.com (T. Mészáros), mihaly.judith@ttk.mta.hu (J. Mihály), bota.attila@ttk.mta.hu (A. Bóta), miklos.kellermayer@med.semmelweis-univ.hu (M.S.Z. Kellermayer), jszebeni2@gmail.com (J. Szebeni).

1 These authors contributed equally to this work.
}

which extends their lifetime in the body and results in doseindependent pharmacokinetics (except for very low doses) [2,3]. Liposome therapy made it possible to increase the bioavailability of drugs that are poorly absorbed (like amphotericin-B), to reduce side effects of highly cytotoxic anti-cancer agents (such as doxorubicin) and to open new routes to generate site-selective effect (e.g.: photodynamic therapy in macular degeneration) [4]. However, new benefits may be accompanied by new risks: e.g., it turned out that liposomes can induce complement activation related pseudoallergy (CARPA), a new type of drug-induced acute immune toxicity [5]. It is suspected that one of its causes could be the presence of liposomal aggregates in the formulated product [6].

Here we report that ammonium sulfate (AS) and other kosmotropic agents may elicit the aggregation and even the fusion of PEGylated liposomes. The aggregates are formed by hydrophobic interactions due to the solvophobic effect of increasing salt concentration [7]. This phenomenon is similar to the salting-out method 
Table 1

Liposome compositions.

\begin{tabular}{ll}
\hline PEG molar\% & molar ratio (Cholesterol:mPEG:HSPC) \\
\hline 0 & $38.4: 0: 61.6$ \\
2 & $38.7: 2: 59.3$ \\
5 & $38.4: 5: 56.6$ \\
10 & $38.4: 10: 51.6$ \\
\hline
\end{tabular}

regularly used in protein fractionation and purification [8,9]. An understanding of the mechanism of aggregate formation could provide invaluable information for successful drug formulations where liposome aggregation could be either prevented or controlled.

\section{Materials and methods}

\subsection{Materials}

Cholesterol, mono PEGylated 1, 2-distearoyl-glycero-3phophoethanolamine (mPEG-2000-DSPE), and hydrogenated soybean phosphatidylcholine (HSPC) were obtained from Lipoid GmbH (Ludwigshafen, Germany). Ethanol, isopropanol, histidine, sucrose, ammonium sulfate (AS), sodium sulfate, magnesium sulfate, sodium citrate, magnesium chloride, guanidine chloride $(\mathrm{GdmCl})$ were purchased from Sigma Aldrich Kft. (Budapest, Hungary). Salsol infusion (TEVA Hungary Zrt., Debrecen, Hungary) was obtained from the University Pharmacy, and purified water was produced by a Milli-Q Integral 3 Water Production Unit (Merck Millipore, Billerica, MA, USA).

\subsection{Liposome preparation}

Liposome suspension with a lipid and buffer composition similar to the FDA-approved and marketed Doxil ${ }^{\circledR}$ was prepared with the extrusion method [10]. The lipid composition was cholesterol, mPEG and HSPC (see molar ratios in Table 1). The lipids were solubilized in ethanol-isopropanol mixture (50:50), then the solution was added dropwise to $0.25 \mathrm{M}$ AS containing $0.9 \%$ saline (SALSOL) solution. Large, heterogeneous lipid particles were extruded four times through $80 \mathrm{~nm}$ Whatman Nuclepore (Track-Etched Membranes) membrane filters (Whatman, Maidstone, UK) by means of a Lipex ${ }^{\mathrm{TM}}$ Extruder (Northern Lipids Inc., Burnaby, B.C. Canada) at 50 bar and $70^{\circ} \mathrm{C}$ to achieve uniform particle size distribution. The liposomes were then dialyzed against $10 \mathrm{mM}$ histidine buffer $(\mathrm{pH}=7.5)$ containing $10 \mathrm{w} / \mathrm{w} \%$ sucrose to remove AS and organic solvents. The total phospholipid concentration was approximately $15.9 \mathrm{mg} / \mathrm{ml}$ (cca. $21.4 \mathrm{mM}$ ). The liposomal stock solutions were stored at $4{ }^{\circ} \mathrm{C}$ protected from light and used within 2 weeks. The stock solution was further diluted as dictated by the experiments. The degree of dilution and corresponding lipid concentrations are given in the text and figure captions.

\subsection{Mixing PEGylated liposomes with different salts}

For turbidimetry, light scattering and zeta potential experiments $20 \mu \mathrm{l}$ of PEGylated liposomes were mixed with $980 \mu \mathrm{l}$ salt solution of appropriate concentration (50x dilution). For phase contrast and atomic force microscopy experiments PEGylated liposomes were diluted either $200 x$ or $500 x$ with salt solution of appropriate concentration. For $0 \mathrm{M}$ concentration physiological saline solution (Salsol) was used for dilution. The actual salt and lipid concentrations are given in the text and figure captions.

\subsection{Dilution of precipitates for fusion and aggregation-reversibility studies}

$500 \mu \mathrm{l}$ of PEGylated liposomes were mixed with $500 \mu \mathrm{l}$ of AS solution to produce a stock of precipitated samples of the desired AS concentration (from $0.8 \mathrm{M}$ to $2.0 \mathrm{M}$ ). After $15 \mathrm{~min}$ incubation time, $20 \mu \mathrm{l}$ of these stocks was mixed to $980 \mu \mathrm{l}$ AS solutions of appropriate concentrations (down to $0.1 \mathrm{M}$ ). The actual salt and lipid concentrations are given in the text and figure captions.

\subsection{Turbidimetry}

The aggregation of PEGylated liposomes was followed by measuring the apparent optical density of the solution. Briefly, a $4 \mu \mathrm{l}$ sample of the well-vortexed solution was pipetted onto the pedestal of a NanoDrop 2000 UV-vis spectrophotometer (Thermo Scientific Ltd., Wilmington, DE), and the optical density was recorded at $250 \mathrm{~nm}$. Because the lipid concentration was kept constant, an increase in optical density corresponds to an increase in light scatter caused by the appearance of larger particles due to aggregation. For comparability, identical lipid concentrations were used in the different samples.

\subsection{Dynamic light scattering measurement}

The size distribution of liposomes and aggregates were characterized by dynamic light scattering (DLS) on a Zetasizer Nano $S$ instrument (Malvern Instruments Ltd, Malvern, UK). From the intensity fluctuations of a 633-nm laser light scattered at high angle from the freely moving suspended particles their diffusion constant was obtained. Size distribution was calculated by using the StokesEinstein equation by the built-in algorithms of the instrument's software. Light scattering was measured at $25 \pm 1^{\circ} \mathrm{C}$. Z-average values are displayed throughout the article, which represent the primary and most stable parameter produced by DLS technique [11] and recommended for quality control reports (ISO 22412:2008). Zaverage values represent a good approximation of hydrodynamic diameter of well dispersed particles with monomodal size distribution (index of polydispersity typically lower than 0.1 ) and thus are well applicable for PEGylated vesicles. The Z-average, however, does not reflect the real size of precipitated samples that are often heterogeneous in size and may be irregularly shaped. In the latter case Z-average was used only for rough estimation of particle size, which enabled us to follow liposome aggregation without exact determination of aggregate dimensions. Since different batches of PEGylated liposomes were used in the different experiments, minor variations are seen in the average size of control vesicles.

\subsection{Zeta potential measurements}

PEGylated liposomes were diluted with AS solution, and $750 \mu \mathrm{l}$ of this mixture was injected carefully into folded capillary cells (PCT Kft., Mosonmagyaróvár, Hungary) to avoid bubble formation. Zeta potential measurements were performed by using a Zetasizer Nano ZS equipment (Malvern Instruments Ltd., Worcestershire, UK) in which particle velocity is measured according to a light scattering technique based on Doppler effect evoked by a pair of mutually coherent laser beams ( $4 \mathrm{~mW}$, He-Ne laser at $633 \mathrm{~nm})$. From the autocorrelation function of the scattered light intensity the electrophoretic mobility and, via the Henry equation, the zeta potential are calculated. Measurements were carried out in triplicates at $25^{\circ} \mathrm{C}$. 


\subsection{Analysis of zeta potential data}

Binding of ions to liposomal surface and the concomitant change of surface potential can be described well by Langmuir-Freundlich isotherm [12].

$\zeta=\zeta_{0}+\Delta \zeta_{\max } \cdot \frac{(K \cdot c)^{n}}{1+(K \cdot c)^{n}}$

where $\zeta$ is the measured zeta potential, $\zeta_{0}$ is the zeta potential at zero AS concentration, $\Delta \zeta_{\max }$ is the maximal change of zeta potential, $\mathrm{K}$ is the binding constant, $\mathrm{c}$ is the ligand concentration and $n$ is the index of heterogeneity describing the cooperativity of ion binding.

\subsection{Atomic force microscopy and image analysis}

Atomic force microscope (AFM) images were recorded with a Cypher instrument (Asylum Research, Santa Barbara, CA) by scanning the samples in fluid with a gold-coated silicon nitride cantilever (Olympus Biolever, A lever, typical spring constant: $30 \mathrm{pN} / \mathrm{nm}$ ). $100 \mu \mathrm{l}$ sample was applied on a cleaned borosilicate glass coverslip and incubated in a vapor chamber at $23 \pm 1^{\circ} \mathrm{C}$. Noncontact-mode images were recorded at a linescan rate of $0.5-1 \mathrm{~Hz}$. All measurements were carried out at $28 \pm 1^{\circ} \mathrm{C}$. Images were analyzed by using the built-in algorithms of the AFM driving software (IgorPro, WaveMetrics, Inc., Lake Oswego, OR).

\subsection{Phase contrast microscopy}

Micrographs were recorded with a Nikon Eclipse Ti-U inverted microscope (Auro-Science Kft., Budapest, Hungary) equipped with a uEye UI 1220 LE digital camera (IDS Imaging Development Systems $\mathrm{GmbH}$, Obersulm, Germany) using a 40x Nikon S Planfluor phase contrast objective.

\subsection{Infrared spectroscopy}

ATR-FTIR spectra were collected with a Varian 2000 FTIR Scimitar Series (Varian Inc., Paolo Alto, CA) spectrometer equipped with a 'GoldenGate' (Specac Ltd., London, UK) single reflection diamond ATR accessory. The measurements were performed at room temperature: $3 \mu$ l sample was mounted on the top of the diamond ATR crystal and a cap was used to avoid sample drying; 128 scans were collected at a resolution of $2 \mathrm{~cm}^{-1}$. ATR correction was executed after each data collection. All spectral manipulations, including subtractions and spectral deconvolutions were performed by using the GRAMS/32 software package (Galactic Industries Incorporation, USA). Band positions for curve fitting were determined using the second derivative. Band shapes were approximated by Lorentzian functions. The intensities and the bandwidth of each component were allowed to vary until the minimal $\chi^{2}$ parameter was reached. After the fitting procedure, the relative contribution of a particular component was calculated from the integrated areas of the individual components.

\section{Results and discussion}

\subsection{PEGylated liposomes can be precipitated by ammonium sulfate}

The addition of AS to PEGylated liposomes in few molar concentration initiated the rapid increase of opacity of the sample. To assess the magnitude of liposome precipitation, we measured the turbidity of liposomal suspensions at 0-2 M AS concentration. According to the turbidity vs. ammonium-sulfate concentration

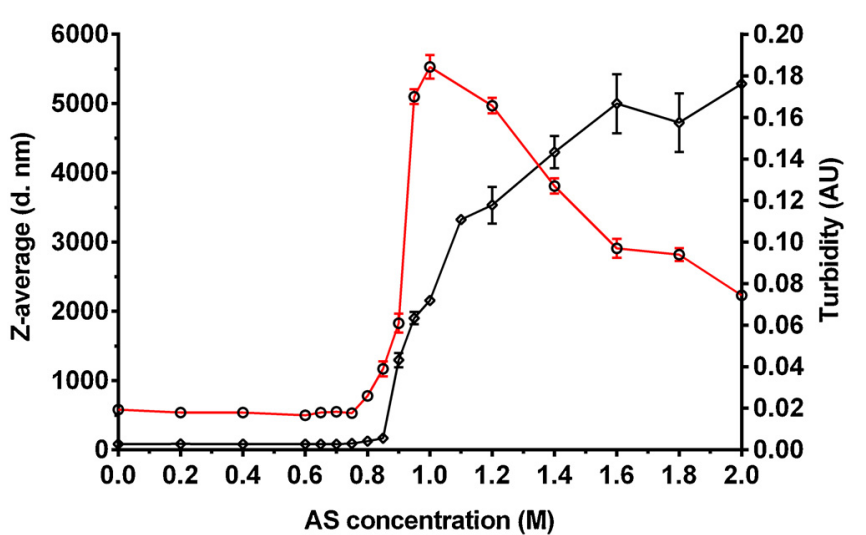

Fig. 1. Turbidity (black line) and average particle diameter (red line) of liposomal suspensions as a function of ammonium sulfate (AS) concentration. Average \pm SD values of three independent measurements are shown. Lipid concentration was kept constant throughout the measurement (50x dilution, $0.318 \mathrm{mg} / \mathrm{ml}$ ). (For interpretation of the references to colour in this figure legend, the reader is referred to the web version of this article.)

curve (Fig. 1), no precipitation occurs below an AS concentration of $0.75 \mathrm{M}$. Above $0.75 \mathrm{M}$, turbidity rises abruptly then levels off above $1 \mathrm{M}$ to a value an order of magnitude greater than in the absence of AS. From this curve we concluded that precipitation began somewhere between 0.75 and $0.8 \mathrm{M}$ AS concentration. The fall of the turbidimetry curve above $1 \mathrm{M}$ AS concentration may be explained by increasing heterogenity of the system, i.e. the formation of a lipid-rich precipitate and a lipid-poor aqeous phase.

We hypothesized that the abrupt rise in turbidity was related to a size increase due to the aggregation of the vesicles. To assess the size of the precipitates as a function of AS concentration, dynamic light scattering measurements were carried out (Fig. 1). Below a concentration of $0.8 \mathrm{M}$ the mean particle size varied between $83.2 \mathrm{~nm}$ and $91.5 \mathrm{~nm}$ independently of the AS concentration. Upon increasing the AS concentration further, however, size increased abruptly to $\sim 2000 \mathrm{~nm}$ in the range of $0.8-1 \mathrm{M}$ then more slowly above $1 \mathrm{M}$. Upon reaching an AS concentration of $2 \mathrm{M}$ the mean particle size exceeded $5000 \mathrm{~nm}$. Notably, particle size is approximated with the Z-average value which is calculated by assuming spherical geometry and monomodal size distribution. In case of aggregation, particle shape is likely to deviate from spherical, which, together with growing polydispersity indices measured above $0.75 \mathrm{M}$ AS (data not shown) means that the Z-average parameter may correspond only to an approximation, within an order of magnitude, of the average particle diameter. The results of the turbidimetry and dynamic light scattering measurements lead to similar conclusion: precipitation begins upon reaching an AS concentration threshold (0.7-0.8 M), then progressively larger aggregates are formed upon incerasing the AS concentration further. Since precipitation takes place instantenously upon mixing the PEGylated liposomes with AS, it is rather difficult to follow aggregation kinetics. Size increases rapidly and considerably in the first minute needed to set up a DLS measurement. After this lag time a further continous increase of size was observable (Fig. S1), but exact rate and kinetics could not been determined.

To reveal the microscopic details of the precipitation process and assess whether vesicle aggregation takes place indeed, morphological measurements were carried out. Phase contrast microscopy of precipitated samples showed branching objects of irregular shapes apparently formed of smaller clusters (Fig. 2) which resembled electron micrographs of liposomal aggregates seen earlier [13].

To resolve the ultrastructure of aggregates and to follow their formation we imaged liposomes with atomic force microscopy (AFM) at various AS concentrations (Fig. 3). At 0 and $0.7 \mathrm{M}$ AS 


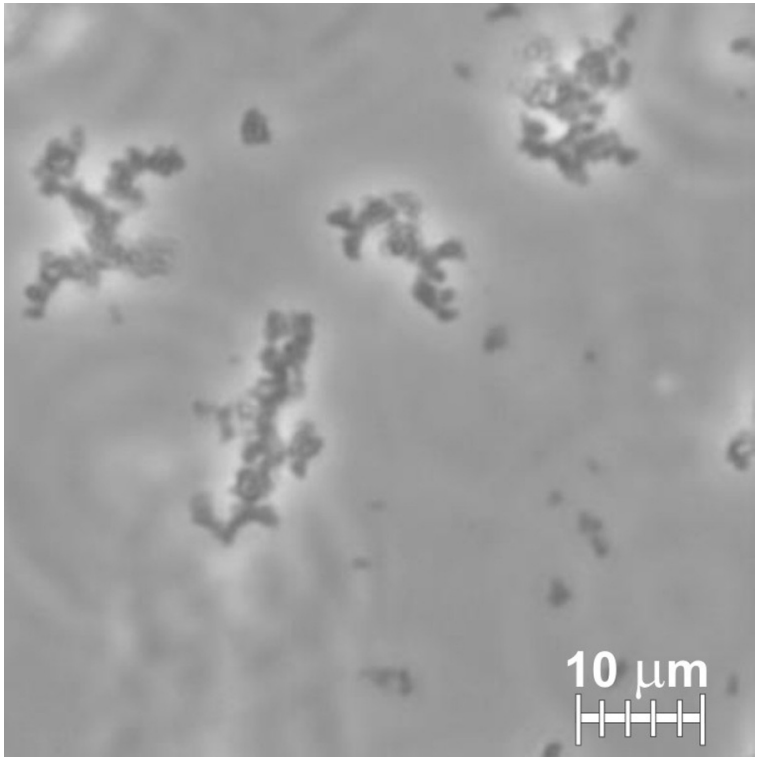

Fig. 2. Phase contrast micrograph of liposomal aggregates in $1 \mathrm{M}$ AS. Large branching objects and smaller aggregates are seen (200x dilution, $0.0795 \mathrm{mg} / \mathrm{ml}$ lipid concentration).

individual, interaction-free liposomes were observed. At an AS concentration of $0.8 \mathrm{M}$ many liposomes were observed in linear assemblies, pointing at the onset of aggregation. At an AS concentration of $0.9 \mathrm{M}$, large aggregates were clearly seen. In $0.8 \mathrm{M}$ AS (Fig. 3C), besides the vesicles flat patches with a smooth surface and a topographical height of 5-7 nm were observed. We identify them as bilayers, although they are somewhat thinner than a lipid bilayer covered with a PEG polymer brush on both sides (approx. $12 \mathrm{~nm}$ ) calculated with a different method $[14,15]$. The bilayer patches probably emerge because as osmolality increases, vesicles exhibit a greater propensity to burst on the substrate [16,17]. Patch formation is a general phenomenon characteristic to liposomal samples. Patches of varying sizes are found in almost all liposomal AFM images throughout the corresponding literature [18-20] and also in Fig. 4. Interestingly, patch formation appears to depend on the AS concentration as evidenced by our results shown in Fig. 3. While patch formation is only sporadic at lower AS concentrations, it becomes pronounced above $0.8 \mathrm{M}$ (Fig. 3C), and at $0.9 \mathrm{M}$ most of the substrate is covered with a confluent supported lipid bilayer (see the background of clusters and vesicles in Fig. 3D). Liposome clusters observed in $0.9 \mathrm{M} \mathrm{AS}$ (Fig. 3D-F) have diverse sizes varying from few hundred nm to few $\mu \mathrm{m}$, which is in an order-of-magnitude correlation with the DLS data (see Fig. 1). Note that the irregular vesicle shape might be the result of imaging artifacts and not exquisitely of liposomal shape transformations. Upon raising the AS concentration above $0.8 \mathrm{M}$ imaging became difficult, which is most likely due to the presence of large, soft aggregates incompletely immobilized on the surface. Several attempts have been made to image samples at even higher AS concentrations to find larger aggregates, but unsuccessfully.

\subsection{Aggregation may promote vesicle fusion}

To test whether liposome aggregation is followed by fusion and to determine the threshold concentration of fusion, liposomal samples were precipitated in various concentrations of AS, then $15 \mathrm{~min}$ later diluted to $0.1 \mathrm{M}$ AS. DLS data showed no considerable change of average size at 0.8 and $0.9 \mathrm{M}$ precipitating concentration. A slight increase was observed at 1.0 and $1.1 \mathrm{M}$ followed by a more signif-
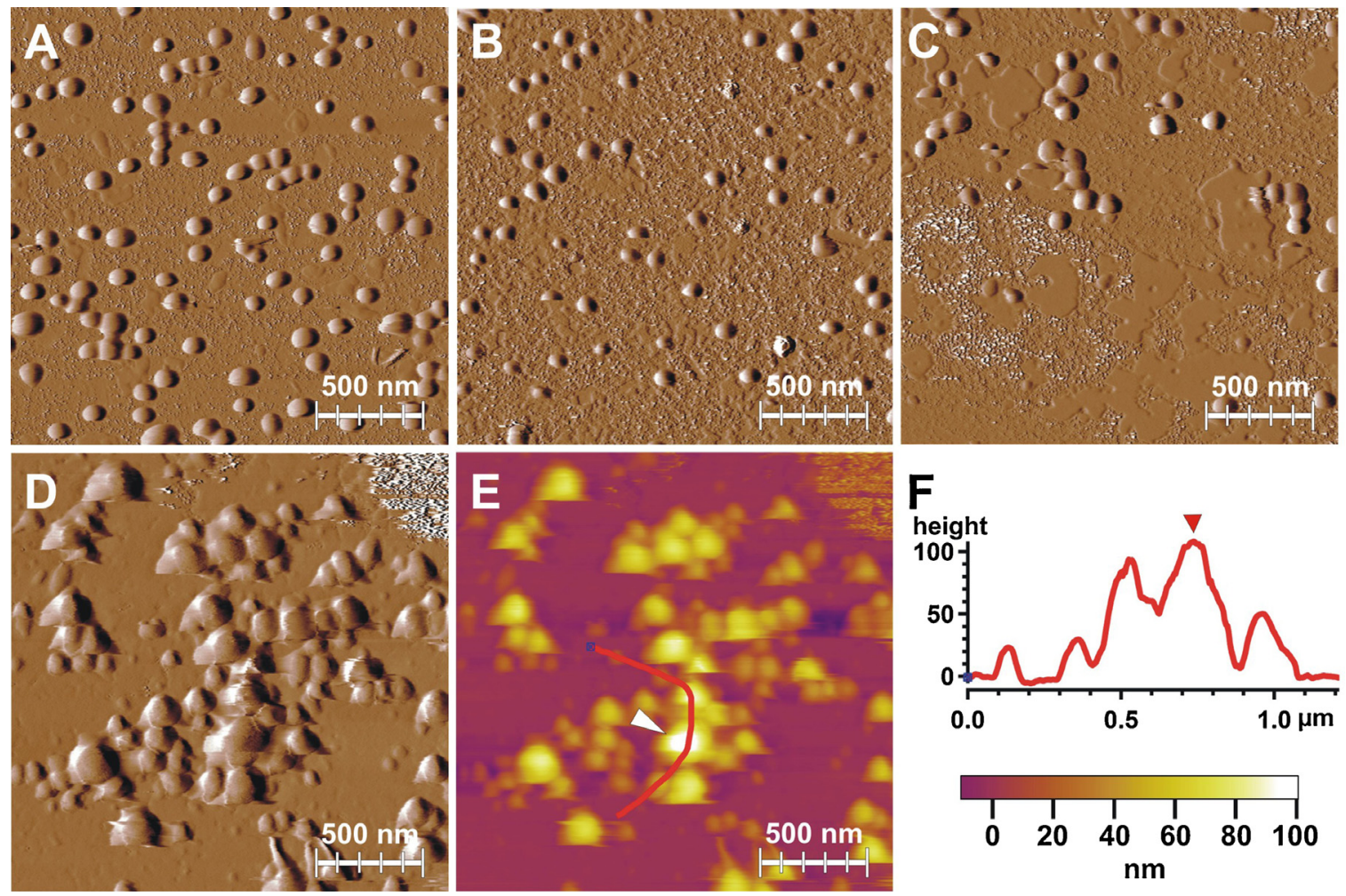

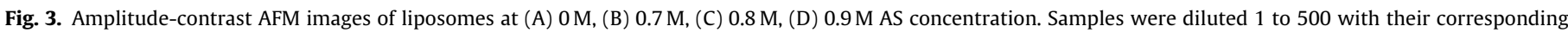

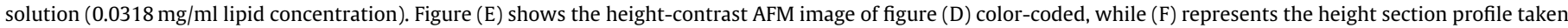

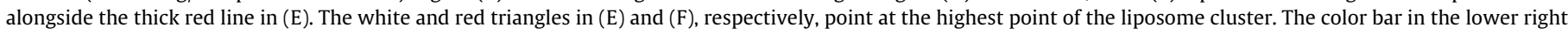
corner displays the height scale of figure (E). (For interpretation of the references to colour in this figure legend, the reader is referred to the web version of this article.) 

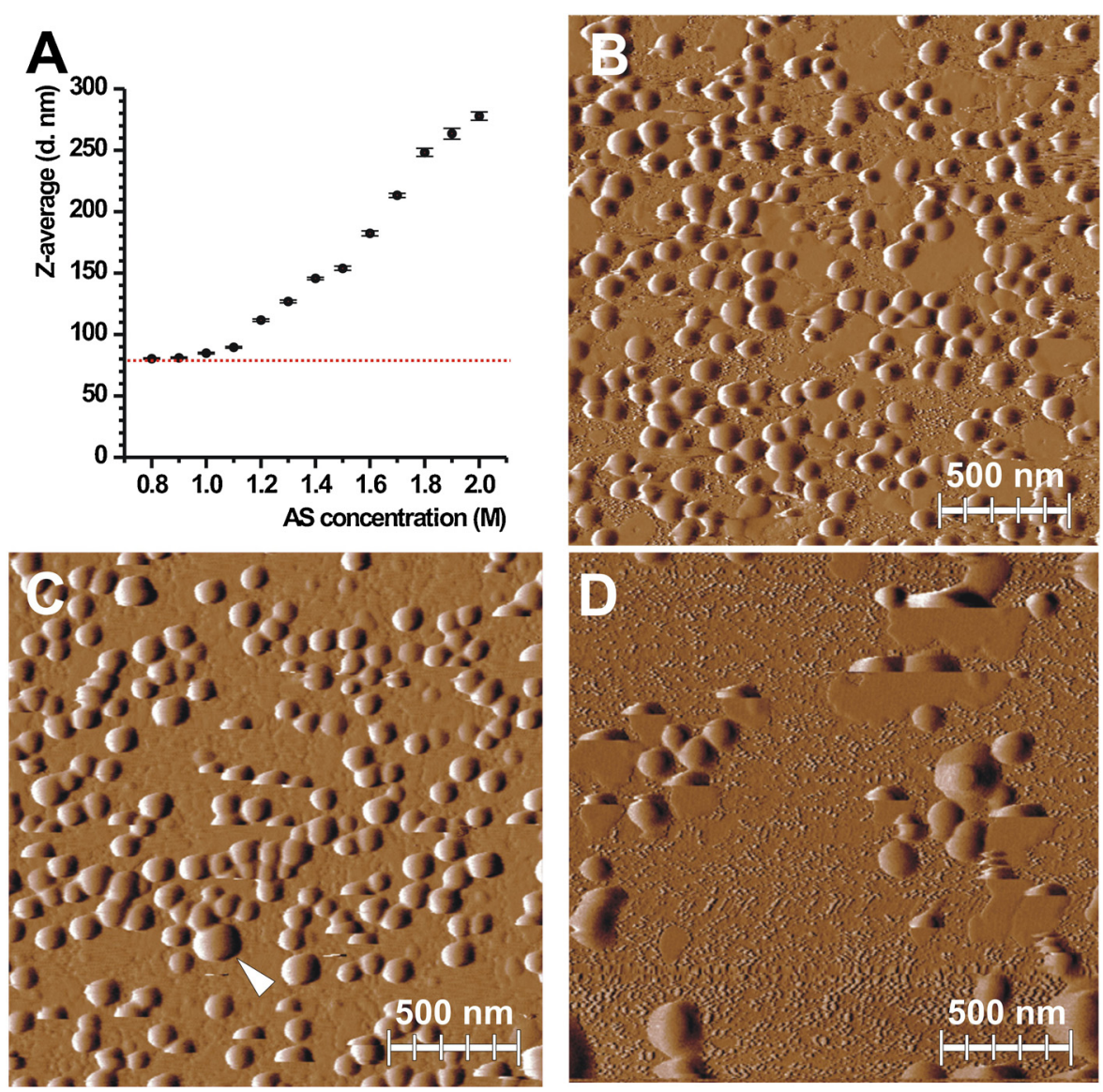

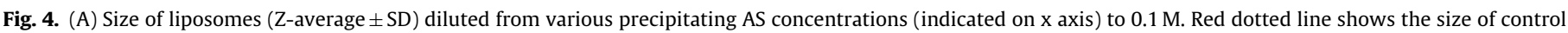

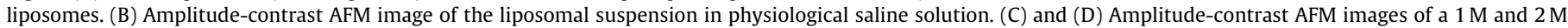

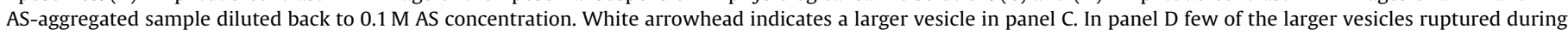

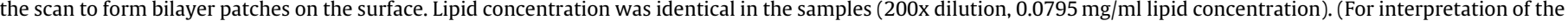
references to colour in this figure legend, the reader is referred to the web version of this article.)

icant and monotonous increase from 1.2 M (Fig. 4A). AFM images of these samples revealed that the increase of average size may be attributed to the occurrence of fused liposomes. Both the size and relative amount of fused vesicles increased with AS concentration: at $1 \mathrm{M}$ liposomes larger than the average appeared sporadically (Fig. 4C), while at $2 \mathrm{M}$ even larger vesicles with an irregular shape became dominant (Fig. 4D). The findings are in accord with size distribution shown in Fig. S2, the averages of which are shown in Fig. 4A. Up to $1.1 \mathrm{M}$ a moderate, then from $1.2 \mathrm{M}$ a more significant upward shift is seen due to the appearance of larger objects. In addition, from $1.5 \mathrm{M}$ a minor population of several-micron-diameter particles appeared which was not observed in AFM images possibly due to their small number.

Taken together, PEGylated liposomes may not only aggregate but also fuse upon AS addition. The rate of fusion is concentration dependent, and the threshold concentration is $1 \mathrm{M}$ at the employed incubation time. This also means that AS-driven aggregation of the vesicles can be reversed depending on AS concentration and incubation time. To disrupt aggregated liposomes they should be diluted well below the AS concentration at which they started to precipitate (Fig. S3).

\subsection{Precipitation does not depend on surface-charge properties}

Physical stability of colloidal vesicles depends largely on their surface charge density, which is approximated with the electric potential difference (or zeta potential) between the bulk solution and the slipping plane of ions associated to the vesicular surface.
It is widely accepted that a zeta potential lower than $30 \mathrm{mV}$ makes colloidal dispersions prone to aggregation [21]. Since the surface charge properties and thus physical stability of liposomal vesicles can be largely affected by ions [22], modification of the liposomes' zeta potential by AS addition might be a key factor in evoking aggregation. To assess the charge-modulating effect of AS on PEGylated vesicles, zeta potential measurements were carried out.

Zeta potential of the control sample was $-71.7 \pm 2.5 \mathrm{mV}$, which implies a strong negative surface potential and corresponds to an extremely high colloidal stability [21]. Adding AS led to a massive increase of the zeta potential value at even relatively low concentrations (Fig. 5), which may be explained by the association of $\mathrm{NH}_{4}^{+}$ ions to the originally negative vesicular surface. Coordination of $\mathrm{NH}_{4}{ }^{+}$cations to the ether oxygens was proposed by Bailey and Callard [23]. An alternative explanation is that structural modifications of the charge-altering PEG chains (discussed later) may have led to the observed charge increase.

The Langmuir-Freundlich isotherm fits well to the data points suggesting monolayer absorption to a heterogeneous surface. The adsorption constant of $1001 \mathrm{M}^{-1}$ points at outstanding affinity of $\mathrm{NH}_{4}{ }^{+}$ions to the PEG-covered surface and 0.73 as index of heterogenity indicates negative cooperativity of the ions.

The pronounced surface-charge altering effect of AS levels off at as low concentration as about $0.1 \mathrm{M}$, which is nearly an order of magnitude lower than the threshold concentration for aggregation. Furthermore, no considerable change of zeta potential is seen reaching the concentration regime of aggregation (above $0.75 \mathrm{M}$, 


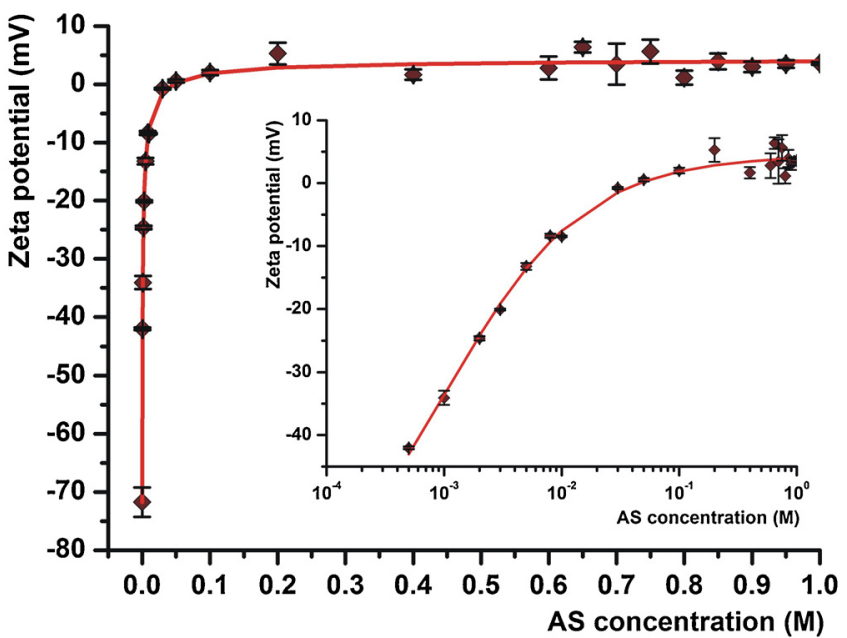

Fig. 5. Zeta potential of PEGylated liposomes as a function of AS concentration. Red line shows the Langmuir-Freundlich isoterm fitted to the data. Zeta potential was measured in triplicates, error bars correspond to standard deviation. Zeta potential at 0 AS concentration was $-71.7 \pm 2.5 \mathrm{mV}$. Inset shows the data plotted on a logarithmic $x$ axis. Dilution: $50 x(0.318 \mathrm{mg} / \mathrm{ml}$ lipid concentration). (For interpretation of the references to colour in this figure legend, the reader is referred to the web version of this article.)

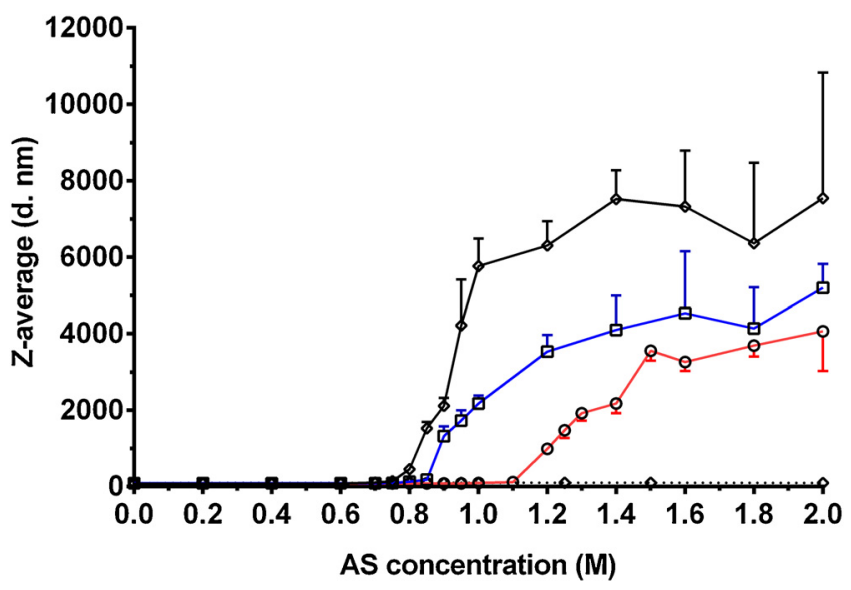

Fig. 6. Effect of PEG concentration on the AS concentration dependence of liposomal aggregation. Dilution: $50 \mathrm{x}(0.318 \mathrm{mg} / \mathrm{ml}$ lipid concentration). Broken line: $0 \% \mathrm{mPEG}$; red line: $2 \%$ mPEG; blue line: 5\% mPEG; black line: 10\% mPEG. (For interpretation of the references to colour in this figure legend, the reader is referred to the web version of this article.)

see Fig. 1). These together suggest that the AS mediated increase of zeta potential is not the mechanism behind liposome aggregation.

\subsection{Precipitation is PEG-related}

To elucidate the role of PEG chains in liposome aggregation, liposomes with different amounts of PEG chains on their surface were produced and mixed with AS. DLS data revealed that conventional liposomes (i.e., ones without PEGylation) showed no size increase up to $2 \mathrm{M} \mathrm{AS}$ (Fig. 6). This indicates that no precipitation takes place in the absence of the PEG brush on the liposomal surface and highlights that the aggregation evoking effect of AS is mediated via the PEG polymer layer. Upon increasing the PEG coverage from 2 to $10 \mathrm{M} \%$, the precipitation curves shifted to the left, meaning that lower concentrations of AS were enough to elicit aggregation. It again underpins that precipitation is PEG-related. Considering that modification of surface charge does not affect the aggregation of liposomes (see above), we hypothesize that some structural transitions of the surface polymer chains must have led to the higher propensity for aggregation.

\subsection{AS dehydrates PEG chains}

Considering that precipitation is related to the presence of PEG, it is plausible that AS, which is a kosmotropic agent [24,25] dehydrated PEG polymers, leading to aggregation of the PEG-covered vesicles through hydrophobic interactions. To estimate the hydration level of the PEG layer, attenuated total reflection Fourier transform infrared spectroscopy (ATR-FTIR) combined with curve fitting procedure was applied. The method was proposed by Varga et al. to characterize the PEG-layer of stealth liposomes based on the ratio of trans and gauche conformations of $\mathrm{C}-\mathrm{O}-\mathrm{C}$ groups of $\mathrm{PEG}$ chain [26]. The complex stretching vibration $\mathrm{C}-\mathrm{O}-\mathrm{C}$ band around $1100 \mathrm{~cm}^{-1}$ of the PEG chain can be decomposed into five band components due to non-interacting vibrations. The band around $1093 \mathrm{~cm}^{-1}$ is related to $\mathrm{C}-\mathrm{O}-\mathrm{C}$ groups of PEG in trans conformation, while the one around $1113 \mathrm{~cm}^{-1}$ belongs to the gauche conformation relative to $\mathrm{C}-\mathrm{C}$ bond [27]. The two extreme components with smaller intensities around 1139 and $1029 \mathrm{~cm}^{-1}$ can be assigned to $\delta\left(-\mathrm{CH}_{2}-\right)$ deformation and $v(\mathrm{C}-\mathrm{C})$ stretching vibrations, respectively. The relatively intense band component around $1068 \mathrm{~cm}^{-1}$ might be related to the $\nu(\mathrm{C}-\mathrm{OH})$ bands. A higher extent of intramolecular $\mathrm{H}$-bonding of the $\mathrm{C}-\mathrm{O}-\mathrm{C}$ groups of the PEG moiety to a neighboring etheric oxygen results in a greater proportion of the more constrained gauche conformer with an appropriate increase of relative intensity. Thus, the ratio of the trans and gauche conformations can be a marker to characterize the PEG layer structure and, indirectly, the hydration state [26].

ATR-FTIR spectra of the PEG-liposomes with 1-2 wt.\% concentration are dominated by the strong water absorption bands; so, as a first step of spectral evaluation the subtraction of water background (AS solution) was performed. Since the $v(\mathrm{C}-\mathrm{O}-\mathrm{C})$ vibration bands overlap with the phosphate stretching vibrations $\left(\nu \mathrm{PO}_{2}^{-}\right)$of the
A

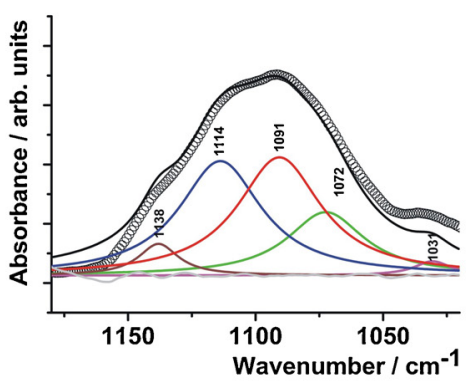

B

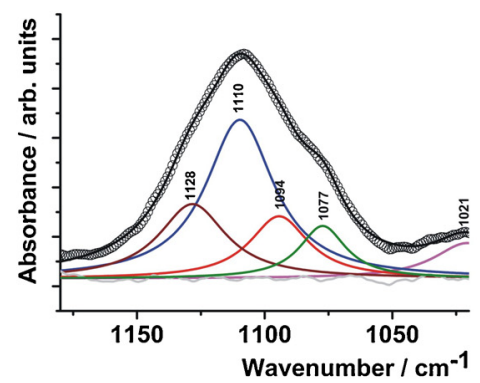

C

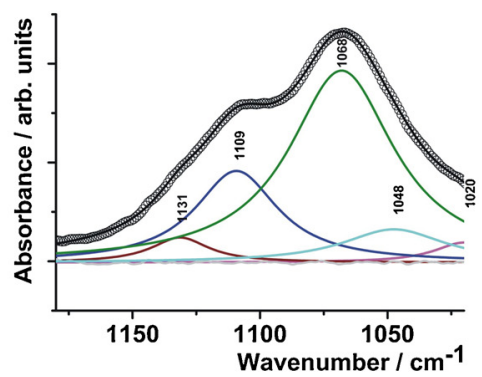

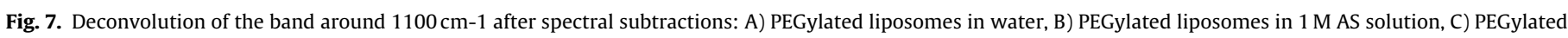
liposomes in $2 \mathrm{M}$ AS solution. Empty circles denote the measured data points, solid lines represent the fitted spectra, the individual band components and the residuals. 


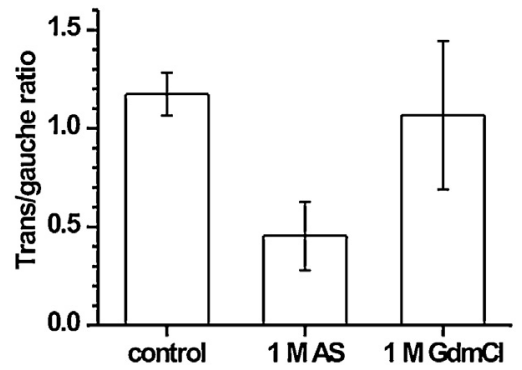

Fig. 8. The trans/gauche ratio of the $v(\mathrm{C}-\mathrm{O}-\mathrm{C})$ band of PEG chains for SSL samples in water, in $1 \mathrm{M}$ ammonium sulfate and in $1 \mathrm{M}$ guanidine chloride. Averages $\pm \mathrm{SDs}$ of three independent measurements are shown. The high standard deviation for guanidine chloride samples might be caused by the difficulty in guanidine chloride background subtraction.

lipid components, the reference spectrum of pure hydrated HSPC (hydrogenated soy phosphatidylcholine, main lipid component for PEGylated liposomes) was carefully subtracted from the spectra of PEGylated liposomes. Typical deconvolutions of the band around $1100 \mathrm{~cm}^{-1}$ after spectral subtraction are presented in Fig. 7 .

The higher the relative intensity of trans conformers, the higher the hydration level of the PEG layer. By adding ammonium sulfate salt ( $1 \mathrm{M}$ concentration) the relative intensity of $v(\mathrm{C}-\mathrm{O}-\mathrm{C})$ in gauche conformation increases (Fig. 8), indicating that the kosmotropic salt reduces the hydration of the PEG polymer chains. As to the higher amount of AS ( $2 \mathrm{M}$ concentration), however, a new band component at $1068 \mathrm{~cm}^{-1}$ dominates the spectrum. This band component might be assigned to $v(\mathrm{C}-\mathrm{OH})$ groups. Interestingly, no band component belonging to $v(\mathrm{C}-\mathrm{O}-\mathrm{C})$ trans component could be deconvoluted. This may indicate a conformational change more pronounced than the trans - gauche variation. Similar phenomenon was observed also for micelles formed by pure DSPE-PEG2000 lipid ( $\sim 10$ wt.\%) in water (unpublished results).

FTIR data support the notion that the mechanism behind aggregation of stealth vesicles might be the reduction of hydration of PEG polymer chains due to kosmotropic effect of AS. This is further underpinned by the observation that a chaotropic salt, guanidine chloride $(\mathrm{GdmCl})$ did not affect significantly the $v(\mathrm{C}-\mathrm{O}-\mathrm{C})$ trans/gauche ratio and thus the hydration level of the polymer (Fig. 8., see spectrum, and deconvolution of the bands in Fig. S4. and Table SI.).

\subsection{Other kosmotropic salts also lead to precipitation of PEGylated liposomes}

We studied the effect of various salts on stealth liposomes to test the hypothesis that PEG-related precipitation of liposomes is not specific for AS but a general kosmotropic phenomenon. All examined kosmotropic salts (sodium citrate, sodium sulfate, magnesium sulfate) led to the precipitation of the vesicles (Fig. S5). By contrast, chaotropic salts (magnesium chloride and guanidine chloride) did not aggregate the liposomes (Fig. S6). The precipitating effect of kosmotropic salts was found to be concentration dependent, and the order of their threshold concentration was the following: sodium citrate< sodium sulfate < magnesium sulfate $<$ ammonium sulfate (Fig. 9). This order is in good accordance with two phase forming capacity of ions in PEG-salt-water systems observed earlier [28].

The surface charge modifying effect of kosmotropic salts levelled off at much lower concentrations than that needed for aggregation (Fig. S7), similarly to what was observed for AS (Fig. 5). The adsorption constants of the ions do not correlate with their precipitating ability. Furthermore not only kosmotropic, but also chaotropic salts, which do not aggregate PEG-liposomes, shifted the

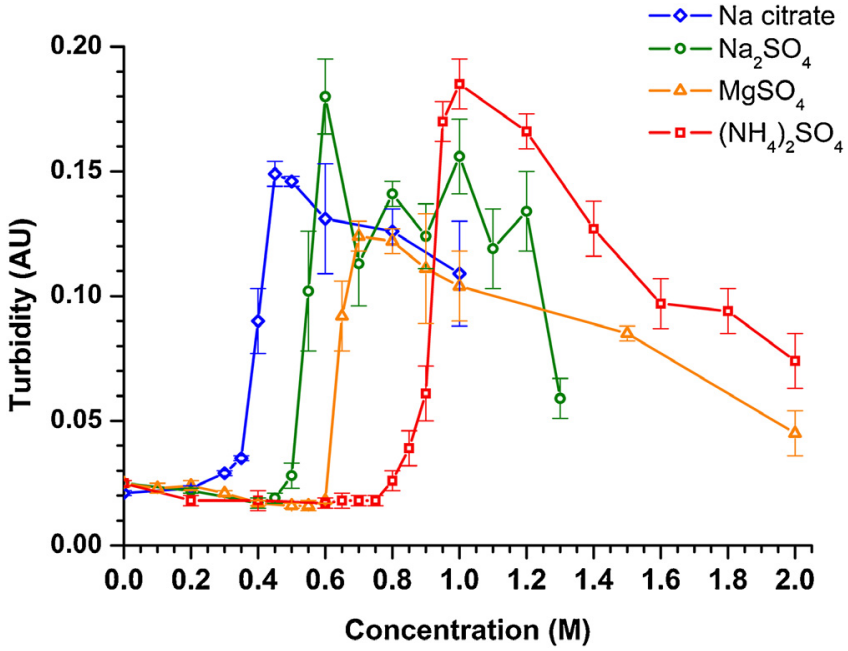

Fig. 9. Turbidity of liposomal suspensions as a function of salt concentration. Average \pm SD values of three independent measurements are shown. PEG concentration was $5 \mathrm{~mol} \%$ and lipid concentration was kept constant throughout the measurement (50x dilution, $0.318 \mathrm{mg} / \mathrm{ml}$ ).

zeta potential of liposomes from strongly negative values to neutral regime (Fig. S7). These together clearly suggest that aggregation phenomenon is not connected to ion adsorption driven surface charge alteration of the vesicles, but to kosmotropic effect.

\section{Conclusions}

Here we demonstrated that ammonium sulfate and other kosmotropic salts have a precipitating effect on PEGylated liposomes. Aggregation takes place above a threshold concentration (Figs. 1,3,6 and 9) and leads to the formation of irregular, micronsized aggregates (Figs. 2 and 3D-F). At certain AS concentrations the process is reversible; aggregates can be fully disintegrated by dilution, but higher AS concentrations may evoke the irreversible fusion of vesicles (Fig. 4). Reduction of surface charge does not have any effect on aggregation of vesicles (Fig. 5). At $0 \mathrm{M} \%$ PEG content no aggregation occurs while the propensity for precipitation increases with PEG coverage in the range of $2-10 \mathrm{M} \%$ (Fig. 6), which clearly shows that AS-driven aggregation of stealth vesicles is related to the PEG chains. AS leads to the dehydration of PEG polymer chains, while $\mathrm{GdmCl}$ does not affect it (Figs. 7 and 8). Other kosmotropic salts (such as $\mathrm{Na}_{3}$ citrate, $\mathrm{Na}_{2} \mathrm{SO}_{4}, \mathrm{MgSO}_{4}$,) also precipitate PEGylated liposomes (Fig. 9), while chaotropic salts (like $\mathrm{GdmCl}$ and $\mathrm{MgCl}_{2}$ ) do not. We propose that kosmotropic salts may induce salting out on the polymer chains promoting their hydrophobic interaction [9] and leading to the separation of the PEG phase together with the coupled liposomes. Reversibility of aggregation is the simple consequence of the reduction of concentration of the kosmotropic agent, similarly to what happens in case of protein purification or during the elution phase of hydrophobic interaction chromatography. The observation that high AS concentration results in rapid fusion of the vesicles may be explained by excessive structural alterations of PEG chains due to their hydrophobic modification. Modification of structure and hydration of PEG chains may lead to steric imbalance of the vesicles as it was shown earlier $[15,29]$.

Since AS (and other kosmotropic agents) may be used during formulation of PEGylated nanoparticles (e.g., remote loading of doxorubicin into liposomes [14]), great care is needed to avoid unwanted aggregation or fusion phenomena, which may take place during either production or storage. There is a hypothesis that complement activation related pseudoallergy (CARPA), observed 
in the clinical practice when PEGylated liposomes are administered intravenously, may be caused by sporadic aggregation or fusion of liposomes [6]. The results presented above may open the route to produce aggregates or fused vesicles in a controlled manner and test their role in pseudoallergic reactions. Reversible aggregation may also be used to separate PEGylated liposomes/nanoparticles from their outer aqueous phase during formulation.

\section{Acknowledgements}

Zeta potential experiments were carried out at Department of Pharmaceutics, Semmelweis University with support of Dr. István Antal and technical assistance of Dr. Viktor Fülöp, for both of which the authors are grateful. We highly appreciate Dr. Pál Grófs help in analysis of zeta potential data.

This work was supported by grants from the Hungarian Science Foundation (OTKA K109480). The research leading to these results has received funding from the European Union's Seventh Framework Program (FP7/2007-2013) under grant agreement no HEALTH-F2-2011-278850 (INMiND) and NMP-2012-309820, (NanoAthero). The authors also acknowledge the financial support by the Hungarian Government National Research, Development and Innovation Fund (TÉT-13-IL-2-2014-0001). The FTIR spectroscopic part was supported by the János Bolyai Research Scholarship of the Hungarian Academy of Sciences (J.M.).

\section{Appendix A. Supplementary data}

Supplementary data associated with this article can be found, in the online version, at http://dx.doi.org/10.1016/j.colsurfb.2016.06. 056.

\section{References}

[1] F.M. Veronese, G. Pasut, PEGylation, successful approach to drug delivery, Drug Discov. Today 10 (2005) 1451-1458

[2] D. Papahadjopoulos, T.M. Allen, A. Gabizon, E. Mayhew, K. Matthay, S.K. Huang, K.D. Lee, M.C. Woodle, D.D. Lasic, C. Redemann, Sterically stabilized liposomes: improvements in pharmacokinetics and antitumor therapeutic efficacy, Proc. Natl. Acad. Sci. 88 (1991) 11460-11464.

[3] T.M. Allen, C. Hansen, Pharmacokinetics of stealth versus conventional liposomes: effect of dose, Biochim. Biophys. Acta (BBA) - Biomembr. 1068 (1991) 133-141.

[4] T.M. Allen, P.R. Cullis, Liposomal drug delivery systems: from concept to clinical applications, Adv. Drug Deliv. Rev. 65 (2013) 36-48.

[5] J. Szebeni, Complement activation-related pseudoallergy: a new class of drug-induced acute immune toxicity, Toxicology 216 (2005) 106-121.

[6] J. Szebeni, P. Bedocs, Z. Rozsnyay, Z. Weiszhar, R. Urbanics, L. Rosivall, R. Cohen, O. Garbuzenko, G. Bathori, M. Toth, R. Bunger, Y. Barenholz, Liposome-induced complement activation and related cardiopulmonary distress in pigs: factors promoting reactogenicity of Doxil and AmBisome, Nanomed.: Nanotechnol. Biol. Med. 8 (2012) 176-184.
[7] P. Cummins, B. O'Connor, Hydrophobic interaction chromatography, in: D. Walls, S.T. Loughran (Eds.), Protein Chromatography, vol. 681, Humana Press, 2011, pp. 431-437.

[8] Interactions of nanoparticles with plasma proteins: implication on clearance and toxicity of drug delivery systems, Expert Opin. Drug Deliv. 8 (2011) 343-357.

[9] M.J. Hey, D.P. Jackson, H. Yan, The salting-out effect and phase separation in aqueous solutions of electrolytes and poly(ethylene glycol), Polymer 46 (2005) 2567-2572.

[10] R.C. MacDonald, R.I. MacDonald, B.P. Menco, K. Takeshita, N.K. Subbarao, L.R. $\mathrm{Hu}$, Small-volume extrusion apparatus for preparation of large, unilamellar vesicles, Biochim. Biophys. Acta 1061 (1991) 297-303.

[11] Dynamic light scattering - common terms defined, in, vol. 2015, www. malvern.com.

[12] X.L.R. Iraolagoitia, M.F. Martini, $\mathrm{Ca}^{2+}$ adsorption to lipid membranes and the effect of cholesterol in their composition, Colloids Surf. B: Biointerfaces 76 (2010) 215-220.

[13] G. Drin, V. Morello, J.-F. Casella, P. Gounon, B. Antonny, Asymmetric tethering of flat and curved lipid membranes by a golgin, Science (New York, N.Y.) 320 (2008) 670-673.

[14] Y. Barenholz, Liposome application: problems and prospects, Curr. Opin. Colloid Interface Sci. 6 (2001) 66-77.

[15] O. Tirosh, Y. Barenholz, J. Katzhendler, A. Priev, Hydration of polyethylene glycol-grafted liposomes, Biophys. J. 74 (1998) 1371-1379.

[16] E. Reimhult, F. Höök, B. Kasemo, Intact vesicle adsorption and supported biomembrane formation from vesicles in solution: influence of surface chemistry, vesicle size, temperature, and osmotic pressure, Langmuir 19 (2003) 1681-1691.

[17] B. Seantier, B. Kasemo, Influence of mono- and divalent ions on the formation of supported phospholipid bilayers via vesicle adsorption, Langmuir 25 (2009) $5767-5772$.

[18] J. Jass, T. Tjarnhage, G. Puu, From liposomes to supported, planar bilayer structures on hydrophilic and hydrophobic surfaces: an atomic force microscopy study, Biophys. J. 79 (2000) 3153-3163.

[19] J. Jass, T. Tjärnhage, G. Puu, Atomic force microscopy imaging of liposomes, in: D. Nejat (Ed.), Methods in Enzymology, vol. 367, Academic Press, 2003, pp. 199-213.

[20] R.P. Richter, A.R. Brisson, Following the formation of supported lipid bilayers on mica: a study combining AFM, QCM-D, and ellipsometry, Biophys. J. 88 (2005) 3422-3433.

[21] T.M. Riddick, Control of Colloid Stability Through Zeta Potential, Zeta-Meter Inc. via Livingston Publishing Company, Wynnewood, 1968.

[22] L. Wu, J. Zhang, W. Watanabe, Physical and chemical stability of drug nanoparticles, Adv. Drug Deliv. Rev. 63 (2011) 456-469.

[23] F.E. Bailey, R.W. Callard, Some properties of poly(ethylene oxide $)^{1}$ in aqueous solution, J. Appl. Polym. Sci. 1 (1959) 56-62.

[24] M.G. Cacace, E.M. Landau, J.J. Ramsden, The Hofmeister series: salt and solvent effects on interfacial phenomena, Q. Rev. Biophys. 30 (1997) 241-277.

[25] J.A. Queiroz, C.T. Tomaz, J.M.S. Cabral, Hydrophobic interaction chromatography of proteins, J. Biotechnol. 87 (2001) 143-159.

[26] Z. Varga, J. Mihály, S. Berényi, A. Bóta, Structural characterization of the poly(ethylene glycol) layer of sterically stabilized liposomes by means of FTIR spectroscopy. Eur. Polym. J. 49 (2013) 2415-2421.

[27] M. Rozenberg, A. Loewenschuss, Y. Marcus, IR spectra and hydration of short-chain polyethyleneglycols, Spectrochim. Acta A: Mol. Biomol. Spectrosc. 54 (1998) 1819-1826

[28] K.P. Ananthapadmanabhan, E.D. Goddard, Aqueous biphase formation in polyethylene oxide-inorganic salt systems, Langmuir 3 (1987) 25-31.

[29] A. Priev, A. Samuni, O. Tirosh, Y. Barenholz, The role of hydration in stabilization of liposomes: resistance to oxidative damage of PEG-grafted liposomes, in: G. Gregoriadis, B. McCormack (Eds.), Targeting of Drugs 6, vol. 300, Springer, US, 1998, pp. 147-167. 